\title{
Análisis de subtipos motores en la enfermedad de Parkinson: Registro Mexicano de Enfermedad de Parkinson (ReMePARK)
}

\author{
Norma L. Alvarado-Franco', Leticia Olguín-Ramírez¹, Robert S. Eisinger², Adolfo Ramírez-Zamora², \\ Amin Cervantes-Arriaga ${ }^{3}$, Mayela Rodríguez-Violante ${ }^{3}$ y Daniel Martínez-Ramírez ${ }^{1}$ \\ ${ }^{1}$ Tecnológico de Monterrey, Escuela de Medicina y Ciencias de la Salud, Monterrey, N.L., México; ${ }^{2}$ Departamento de Neurología, Centro de Trastornos \\ del Movimiento y Neurorestauración, Universidad de Florida, Gainesville, Florida, EE.UU.; ${ }^{3}$ Laboratorio Clínico de Enfermedades Neurodegenerativas, \\ Instituto Nacional de Neurología y Neurocirugía, Ciudad de México, México
}

\begin{abstract}
Resumen
Introducción: La enfermedad de Parkinson (EP) se clasifica de manera empírica en predominio tremorígeno (TD) e inestabilidad postural con trastornos de la marcha (PIGD). Análisis recientes que han utilizado múltiples variables clínicas han encontrado diversos subtipos, los cuales no son fáciles de aplicar en la práctica clínica. Recientemente, empleando un análisis jerárquico de una cohorte americana encontramos cinco subtipos motores y una evolución del subtipo TD al PIGD con el tiempo. La replicación de datos es necesaria para validar el método utilizado y los resultados. Objetivos: Identificar subtipos motores y sus cambios en una cohorte mexicana de EP utilizando un análisis por conglomerados. Métodos: Empleamos el Registro Mexicano de Enfermedad de Parkinson (ReMePARK), del cual obtuvimos todos los puntajes de los componentes motores de la escala unificada de la EP de la Sociedad de Trastornos del Movimiento (MDS-UPDRS), partes II y III, de todas las evaluaciones clínicas. Para identificar subtipos sin utilizar modelos a priori, aplicamos una agrupación jerárquica correlacional de los puntajes de la MDS-UPDRS. De los 197 pacientes analizados, encontramos cinco subtipos (un grupo TD, uno indeterminado [IG] y otro PIGD) y emergieron tres subgrupos del IG (axial, apendicular y rigidez). Después asignamos subtipos para cada visita. Los pacientes con subtipos inconsistentes $(n=77)$ fueron analizados computando frecuencias de subtipos en el tiempo utilizando un intervalo de seis meses para documentar si existía algún cambio de subtipo. Resultados: Las frecuencias de subtipos a los 0-2 años de diagnóstico fueron: 60\% TD, 0\% axial, 14\% apendicular, $5 \%$ rigidez y 21\% PIGD. La regresión linear demostró que los subtipos incrementaron (empeoraron) con la duración de la enfermedad $(p<0.05)$. Los subtipos a los 6-8 años de diagnóstico fueron: TD, 37\%; axial, 8\%; apendicular, 13\%; rigidez, 0\%, y PIGD, 42\%. Conclusiones: Nuestro análisis encontró cinco subtipos motores en una cohorte mexicana. Además, se observó el cambio del subtipo TD al PIGD a los 6-8 años de sintomatología. Nuestros resultados apoyan y sugieren que la duración de la enfermedad puede afectar al subtipo motor en la EP. Los resultados son similares a nuestro estudio previo, en el que se utilizó una cohorte diferente pero con el mismo método analítico sistemático.
\end{abstract}

Palabras clave: Subtipos. Clasificación. Análisis de datos. Progresión. Síntomas motores.

\section{Correspondencia:}

Daniel Martínez-Ramírez

Tecnológico de Monterrey

Escuela de Medicina y Ciencias de la Salud

Av. Ignacio Morones Prieto, 3000 Poniente

Col. Los Doctores

Fecha de recepción: 02/03/2018

Fecha de aceptación: 08/08/2018

C.P. 64710, Monterrey, N.L., México

E-mail: daniel.martinez@medicos.tecsalud.mx

DOI: 10.24875/RMN.M18000002
Disponible en internet: 13-11-2018 Rev Mex Neuroci. 2018;19(5):3-8 www.revmexneuroci.com 1665-5044/@ 2018. Academia Mexicana de Neurología A.C. Publicado por Permanyer México. Este es un artículo Open Access bajo la licencia CC BY-NC-ND (http://creativecommons.org/licenses/by-nc-nd/4.0/). 


\title{
Analysis of Parkinson's disease motor subtypes: Mexican Registry of Parkinson - ReMePARK
}

\begin{abstract}
Background: Parkinson's disease (PD) is commonly classified into tremor dominant (TD) and a postural instability and gait disorder (PIGD) subtypes. Motor subtyping is primarily empirical. Data-driven approaches classify PD into multiple groups. Using hierarchical clustering to analyze the Parkinson's progression markers initiative cohort, we found five motor subtypes and an overall shift away from TD as disease progressed. However, replication is needed to validate results. Objective: To identify motor subtypes and analyze changes in a PD Mexican cohort utilizing a cluster analysis. Methods: We used the Mexican Registry of PD (ReMePARK). Scores to the motor components of the MDS-UPDRS Part II and III at all timepoints were utilized for the analysis. To identify motor subtypes without using a priori models, we applied correlational hierarchical clustering of the MDS-UPDRS scores. Names assigned to these groups were based on the MDS-UPDRS questions they each included. 197 patients were analyzed finding five groups: a TD group, an intermediate group (IG), and a PIGD group. Three subgroups within the IG emerged: axial, appendicular, and rigidity subgroup. We then assigned subtypes for each visit. Patients with inconsistent subtypes were analyzed separately by computing subtype frequencies over time using a bin width of 6 months to produce sample-level averages to document a change in subtype over time. Results: The motor subtype frequencies between 0 and 2 years of diagnosis were as follow: 60\% TD, 0\% axial, 14\% appendicular, $5 \%$ rigidity, and $21 \%$ PIGD between 0 and 2 years of diagnosis. Linear regression showed that subtype increased (worsened) with disease duration $p<0.05$. Of the patients resulting in subtypes that changed at $6-8$ years of diagnosis $(n=77)$, frequencies were as follows: to $37 \%$ TD, $8 \%$ axial, $13 \%$ appendicular, $0 \%$ rigidity, and $42 \%$ PIGD. Conclusion: Five motor subtypes of PD patients resulted from our cluster analysis. Similar than our previous study. The overall shift away from TD was replicated in a cohort with 6-8 years of disease duration. Our results supports that disease duration may affect PD subtype. We replicated our previous study using the same systematic analysis with a different population.
\end{abstract}

Key words: Subtypes. Classification. Cluster analysis. Progression. Motor symptoms.

\section{Introducción}

Aunque la EP fue descrita hace más de 200 años por J. Parkinson, la variabilidad clínica de este complejo síndrome sigue siendo difícil de comprender ${ }^{1}$. Hoy en día necesitamos entender mejor esta variabilidad clínica, ya que resulta fundamental para el pronóstico y el tratamiento de la enfermedad. Además, nos ayudará a definir mejor los criterios de valoración de los ensayos clínicos ${ }^{2}$. Los estudios de subtipificación en la EP pretenden caracterizar con precisión los fenotipos clínicos más comunes y proporcionar un método eficiente y replicable para clasificar al paciente en subtipos $^{3}$. Actualmente no existe un método aceptado universalmente para la clasificación y asignación de subtipos. Esto se debe, en parte, a la falta de replicación de los resultados, los subtipos obtenidos algunas veces no son prácticos en la clínica y el desafío es el de subtipificar a los pacientes en una enfermedad que progresa y cambia con el tiempo 4 .

En la última década se ha incrementado el uso de análisis sistemáticos de bases de datos (minería de datos) para la subtipificación motora y no motora en la $E P^{3}$. Si bien los métodos estadísticos utilizados son novedosos, estas técnicas se ven afectadas por el diseño del estudio (p. ej., estudios transversales), el tamaño de la muestra y la falta de reproducibilidad de los resultados en otras cohortes. Para poder lograr un mejor análisis y en teoría poder abarcar todo el espectro clínico heterogéneo de la EP, se requieren bases de datos grandes con seguimiento longitudinal a largo plazo. A pesar de la gran variedad de subgrupos que se han reportado en la literatura médica ${ }^{5-11}$, existe una designación de subtipos incluida en la mayoría de los estudios y un consenso general de que los pacientes se clasifican en TD y PIGD. Aquellos pacientes que no se ajustan a estos dos tipos se categorizan como en un subtipo indeterminado $(I G)$.

En un estudio reciente analizamos la base de datos del Parkinson's Progression Markers Initiative (PPMI), que incluye pacientes con diagnóstico temprano ${ }^{12}$. En dicho estudio, utilizamos los puntajes motores de la MDS-UPDRS para realizar un análisis de agrupación correlacional jerárquica. El análisis encontró cinco subtipos motores, incluyendo un subtipo de TD, otro de PIGD y un tercero de IG. El subtipo IG se subdividió en tres grupos: uno con predominio axial, otro con predominio apendicular y el último con predominio rigidez. Además, el análisis mostró que, en aproximadamente la mitad de los pacientes, el subtipo TD 
cambiaba lentamente con el paso del tiempo hacia el subtipo PIGD dentro de los primeros cinco años del diagnóstico.

En el presente estudio utilizamos el ReMePARK para analizar al grupo de pacientes con el mismo método con el fin de buscar subtipos motores para determinar su frecuencia. Además, analizamos si estos subtipos motores se mantienen estables o se modifican con la mayor duración de la enfermedad. Con esto también buscamos validar el modelo analítico estadístico en otra población.

\section{Métodos}

El presente es un estudio de cohorte en el que utilizamos la base de datos ReMePARK de pacientes mexicanos con $\mathrm{EP}^{13}$. Los pacientes de dicho registro proporcionaron consentimiento informado previamente. El ReMePARK es un registro nacional longitudinal de pacientes con EP de investigación clínica que proporciona información sobre las características demográficas, clínicas y funcionales de los pacientes. Los criterios de inclusión en el presente estudio fueron: individuos diagnosticados con EP por un neurólogo entrenado en trastornos del movimiento y con una fecha estimada de inicio de los síntomas documentada. Se excluyeron aquellos pacientes que recibieron tratamiento con estimulación cerebral profunda.

Documentamos los puntajes de todas las variables de la parte III (exploración motora) y las variables 10 (temblor), 11 (levantarse de la cama, asiento del coche o sillón), 12 (caminar y equilibrio) y 13 (congelamientos o bloqueos) de la parte II de la MDS-UPDRS durante todas las visitas de todos los pacientes. El método analítico utilizado fue el mismo del trabajo antes mencionado, en el que se empleó una agrupación correlacional jerárquica no a priori de las puntuaciones de las variables mencionadas. El algoritmo sistemático utilizado identifica o agrupa las variables más correlacionadas por grupos utilizando los datos de todas las visitas (puntos de tiempo). Los subtipos se asignaron usando las tasas de las puntuaciones secundarias (puntuación media de los elementos de la MDS-UPDRS dentro de una categoría de subtipo) y los criterios de corte validados (ver sección «Métodos» de Eisinger, et al., 2017) ${ }^{14}$. De los 197 pacientes incluidos en el análisis para determinar agrupaciones, con un promedio de duración de la enfermedad de 4.99 años y un máximo de 16 visitas, se encontraron los siguientes subtipos (agrupaciones): TD, PIGD e IG. Dentro del IG, se identificaron tres subgrupos, que fueron nombrados
Tabla 1. Variables utilizadas para el análisis sistemático obtenidas de la MDS-UPDRS

\begin{tabular}{|l|c|}
\hline Subtipo & Ítem MDS-UPDRS \\
\hline TD & $2.10,3.29$ (cabeza, ESD, ESI, EID, EII) \\
\hline IG & $3.18,3.19$ \\
\hline DAx & $3.23(\mathrm{D}, \mathrm{I}), 3.24(\mathrm{D}, \mathrm{I}), 3.25(\mathrm{D}, \mathrm{I}), 3.26(\mathrm{D}, \mathrm{I}), 3.31$ \\
\hline DAp & 3.22 (cuello, ESD, ESI, EID, EII) \\
\hline RD & $2.11,2.12,2.13,3.27,3.28 .3 .29,3.30$ \\
\hline PIGD & \\
\hline D: derecha; I: izquierda; ES: extremidad superior: El: extremidad inferior.
\end{tabular}

D: derecha; I: izquierda; ES: extremidad superior; El: extremidad inferior.

de acuerdo con las variables de la MDS-UPDRS: dominante axial (DAx), dominante apendicular (DAp) y rigidez dominante (RD) (Tabla 1).

Para analizar posibles cambios de subtipos a lo largo del tiempo, se utilizó el puntaje obtenido durante la evaluación en off medicamentosa de la MDS-UPDRS en todas las visitas clínicas. Para determinar si los pacientes permanecían a lo largo del tiempo en el mismo subtipo clasificado en su primera visita (grupo consistente) o si cambiaban a otro (grupo inconsistente), los subtipos o agrupaciones se calcularon en cada punto de tiempo (visita clínica) para cada paciente, y a los individuos se les asignó el subtipo apropiado, si existía alguno. De los 197 individuos del estudio, observamos que $77(39 \%)$ cambiaban el subtipo a lo largo del seguimiento (grupo inconsistente). En ellos calculamos el percentil 25 de la duración media del tiempo hasta que se produjo un cambio de subtipo. Un total de 120 (60.1\%) pacientes (grupo consistente) mantuvieron un único subtipo durante todo el seguimiento. Este valor se utilizó para definir el tiempo mínimo necesario entre el primer y el último punto de tiempo registrado para definir el subconjunto que presenta un subtipo estable. Analizamos las proporciones medias de diferentes categorías de subtipos a lo largo del tiempo, así como el temblor y las puntuaciones de PIGD. Usamos la prueba de la $\chi^{2}$ y la de regresión lineal con niveles de significancia establecidos en $p=0.05$ usando el software R (www.cran.r-project.org).

\section{Resultados}

De los 197 pacientes, 77 (39\%) se consideraron como grupo inconsistente y 120 (60.1\%) como grupo consistente durante el seguimiento. De los pacientes del grupo inconsistente, las frecuencias de los subtipos 


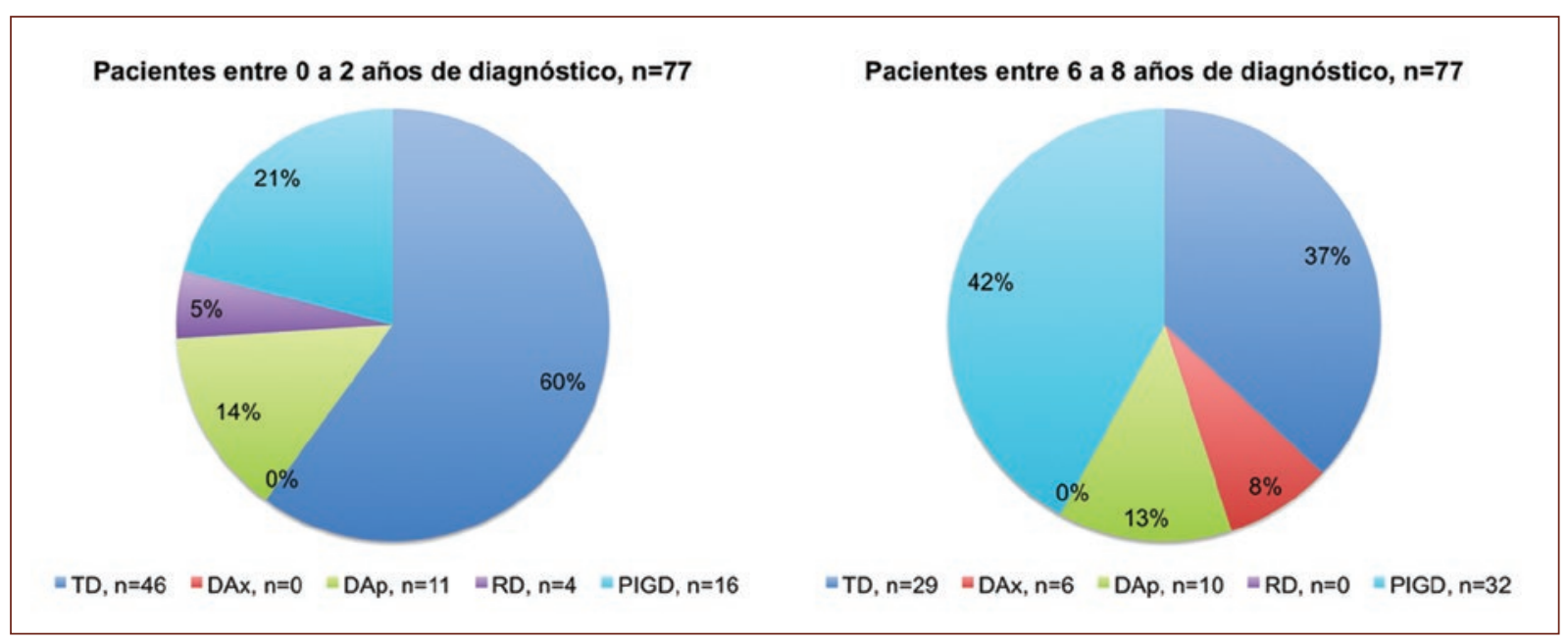

Figura 1. Comparación de frecuencias de subtipos motores al inicio y a varios años del diagnóstico. Se observa un predominio del subtipo motor tremorígeno en los primeros años de diagnóstico (imagen de la izquierda). Al compararse con la imagen de la derecha, a los 6-8 años del diagnóstico, se observa una disminución en la frecuencia del grupo TD (del 60 al 37\%) y un aumento en la frecuencia del subtipo PIGD (del 21 al 42\%). El subtipo PIGD dobla su frecuencia con el tiempo.

a los 0-2 años de diagnóstico fueron las siguientes: el $60 \%(46 / 77)$ de los pacientes estaban clasificados como TD, el 0\% como DAx, el 14\% (11/77) como DAp, el 5\% (4/77) como RD y el 21\% (16/77) como PIGD.

La regresión linear demostró que los subtipos incrementaron (empeoraron) con la duración de la enfermedad $(p<0.05)$. Las frecuencias de los subtipos a los 6-8 años de diagnóstico fueron las siguientes: $37 \%$ (29/77), TD; 8\% (6/77), DAx; 13\% (10/77), DAp; 0\%, RD, y $42 \%$ (32/77), PIGD (Figs. 1 y 2).

\section{Discusión}

En el presente estudio se realizó un análisis por conglomerados de una cohorte longitudinal de pacientes mexicanos (ReMePARK) con EP de 4.99 años de enfermedad. El objetivo principal fue encontrar subtipos motores y ver su progresión en el tiempo. El análisis inicial arrojó los mismos grupos previamente obtenidos de una cohorte americana ${ }^{14}$ : un grupo con TD, otro con PIGD y un tercero indeterminado, que incluye tres subgrupos: DAp, DAx y RD. En la tabla 2 comparamos las frecuencias de subtipos encontradas en el estudio PPMI con las frecuencias del ReMePARK. Con ello demostramos la reproducibilidad de las técnicas sistemáticas anteriores para la subtipificación motora en la EP basadas en evaluaciones motoras simples obtenidas de la MDS-UPDRS. A diferencia de otros estudios ${ }^{3}$, en los que se utilizan variables poco prácticas en la clínica (progresión rápida, inicio joven,

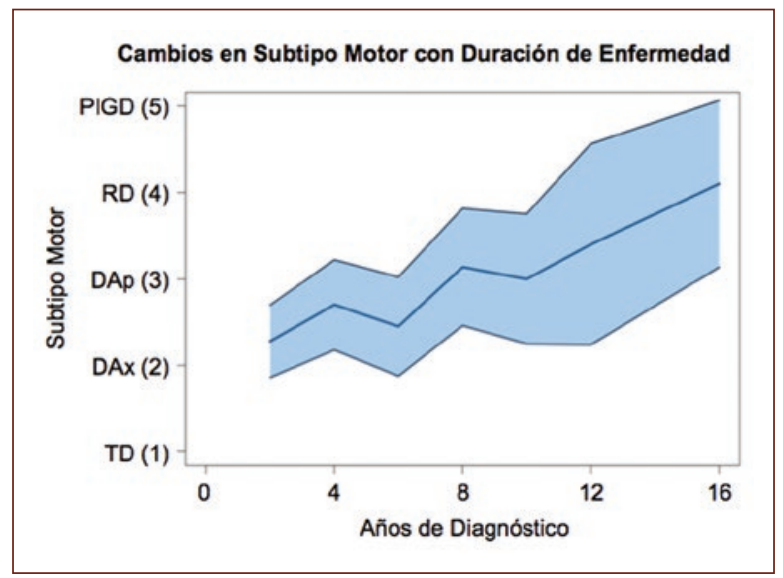

Figura 2. Progresión de los subtipos motores con el tiempo. En esta figura podemos observar la progresión linear de los subtipos motores. Existe una tendencia hacia el cambio de subtipo motor con la duración de la enfermedad.

etc.), la subtipificación que empleamos basada en evaluaciones clínicas y ubicuas obtenidas por la MDS-UPDRS puede simplificar los procedimientos de subtipificación y conducir a categorías con interpretación clínica directa. Es importante tomar en cuenta que con este método los subtipos obtenidos dependen de la evaluación clínica obtenida por el médico examinador. Lo ideal es que sea un médico entrenado en trastornos del movimiento.

También hemos demostrado que con esta técnica de análisis por conglomerados jerárquico no a priori un 
Tabla 2. Comparación de subtipos encontrados por análisis sistemático por conglomerados de la cohorte PPMI con la cohorte ReMePARK

\begin{tabular}{|l|c|c|}
\hline $\begin{array}{l}\text { Duración de la } \\
\text { enfermedad }\end{array}$ & $\begin{array}{c}\text { Cohorte } \\
\text { PPMI (n= 423) }\end{array}$ & $\begin{array}{c}\text { Cohorte } \\
\text { ReMePARK (n = 197) }\end{array}$ \\
\cline { 2 - 3 } & De 0 a 0.5 años & De 0 a 2 años \\
\hline TD & $59.3 \%$ & $60 \%$ \\
\hline PIGD & $21.4 \%$ & $21 \%$ \\
\hline Indeterminado & $3.9 \%$ & $0 \%$ \\
\hline DAx & $11.7 \%$ & $14 \%$ \\
\hline DAp & $3.6 \%$ & $5 \%$ \\
\hline RD & De 4 a 4.5 años & De 6 a 8 años \\
\hline Duración de la & & \\
\hline enfermedad & $40.6 \%$ & $37 \%$ \\
\hline TD & $35.9 \%$ & $42 \%$ \\
\hline PIGD & $10 \%$ & $8 \%$ \\
\hline Indeterminado & $10.9 \%$ & $13 \%$ \\
\hline DAx & $10.9 \%$ & $0 \%$ \\
\hline DAp & & \\
\hline RD & & \\
\hline
\end{tabular}

grupo de pacientes pertenece a subtipos motores cambiantes con el tiempo. En este caso fue del 40\%, similar a lo reportado en el estudio previo de la cohorte PPMI, en el que se encontró que el $45 \%$ de los pacientes permanecían en un subtipo constante a lo largo del tiempo ${ }^{12}$. Sin embargo, en un estudio reciente que empleaba la cohorte de la Universidad de Florida, de más de 2,300 pacientes con EP, encontramos que el $91 \%$ de éstos pertenecían al grupo inconsistente o cambiante (datos aún no publicados). Factores como el número de pacientes, la duración de la enfermedad o el seguimiento a largo plazo pudieran influir en las diferencias reportadas.

También observamos que las proporciones relativas de los subtipos motores encontrados cambiaron de un análisis inicial, con promedio de 0-2 años de diagnóstico, a un análisis posterior, con promedio de 6-8 años de diagnóstico, esto replicando a lo reportado en el estudio previo (Tabla 2). De acuerdo a lo anterior, en un periodo de 6-8 años, las proporciones relativas del subtipo de TD disminuyeron de un 60 al 37\%, las del PIGD aumentaron de un 21 al 42\%, el predominio axial aumentó del 0 al $8 \%$ y el predominio de la rigidez disminuyó del 5 al $0 \%$; sin cambios en el predominio apendicular. En general, los resultados refuerzan la noción previamente propuesta de que hay un subgrupo de pacientes en quienes los subtipos motores de la EP pueden cambiar/ progresar con el tiempo. A este respecto, los métodos de subtipificación basados únicamente en la presentación clínica en el momento del diagnóstico pueden erróneamente clasificar a un individuo durante todo el curso de su enfermedad, en comparación a los enfoques que consideran los cambios en los síntomas motores predominantes a lo largo del tiempo. Los médicos e investigadores que siguen a pacientes con EP durante muchos años deben tener muy presente esta idea.

Este estudio tiene varias limitaciones. En primer lugar, los puntos de tiempo se calculan en referencia a la aparición estimada de los síntomas, en comparación con las fechas exactas del diagnóstico. En segundo lugar, dada la naturaleza retrospectiva del estudio, el número de puntos de datos en cada seguimiento puede ser inconsistente, y disminuyó notablemente con el tiempo. Los estudios prospectivos que siguen grandes cohortes en visitas programadas de manera regular proporcionarán mayor información y una validación crucial de nuestros resultados.

Aún quedan muchas preguntas por contestar como, por ejemplo, ¿cuál es el impacto de factores como la genética, el ambiente, el tratamiento médico o quirúrgico en la asignación del subtipo y la progresión de la enfermedad?, ¿cuáles son las características de aquellos pacientes que permanecen constantes a lo largo del tiempo?, ¿podríamos encontrar factores predictivos para saber quiénes modificarán de subtipo? o ¿cómo podríamos incorporar esta información a los estudios clínicos, principalmente de neuroprotección? Definitivamente, estudios prospectivos futuros podrían explorar factores asociados o terapias que eviten que estos individuos progresen o cambien hacia un subtipo PIGD.

\section{Conclusiones}

Nuestros datos longitudinales replican los resultados obtenidos en el estudio previo encontrando cinco subtipos motores en la EP. También encontramos cambios de subtipos en relación con la duración de la enfermedad. Ello sugiere fuertemente que existe un grupo de pacientes que inicia con un subtipo motor de tipo TD y cambia con el tiempo hacia una designación del subtipo PIGD. Sin embargo, también existe un grupo de pacientes que permanecen en el mismo subtipo a lo largo del tiempo. Con ello validamos el método analítico sistemático por conglomerados utilizado en dos poblaciones diferentes. Este método analítico puede ser de gran utilidad en la práctica clínica. 


\section{Declaración de conflicto de interés}

Los autores declaran que no existen conflictos de interés relevantes para este artículo de revisión.

\section{Fuentes de financiamiento}

No existió una fuente de financiamiento particular para este informe científico.

\section{Agradecimientos}

Nos gustaría agradecer la ayuda de la base de datos ReMePARK, del Instituto Nacional de Neurología y Neurocirugía.

\section{Bibliografía}

1. Rodríguez-Violante M, Cervantes-Arriaga A, Fahn S, Tolosa E. Two-hundred years later: is Parkinson's disease a single defined entity? Rev Invest Clin. 2017;69(6):308-13

2. Berg D, Postuma RB, Bloem B, Chan P, Dubois B, Gasser T, et al. Time to redefine PD? Introductory statement of the MDS Task Force on the definition of Parkinson's disease. Mov Disord. 2014;29(4):454-62.
3. Van Rooden SM, Heiser WJ, Kok JN, Verbaan D, van Hilten JJ, Marinus J. The identification of Parkinson's disease subtypes using cluster analysis: a systematic review. Mov Disord. 2010;25(8):969-78.

4. Simuni T, Caspell-García C, Coffey C, Lasch S, Tanner C, Marek K, et al. How stable are Parkinson's disease subtypes in de novo patients: Analysis of the PPMI cohort? Parkinsonism Relat Disord. 2016;28:62-7.

5. Van Rooden SM, Visser M, Verbaan D, Marinus J, van Hilten JJ. Moto patterns in Parkinson's disease: a data-driven approach. Mov Disord. 2009;24(7):1042-7.

6. Graham JM, Sagar HJ. A data囚driven approach to the study of heterogeneity in idiopathic Parkinson's disease: identification of three distinct subtypes. Mov Disord. 1999;14(1):10-20.

7. Erro R, Vitale C, Amboni M, Picillo M, Moccia M, Longo K, et al. The heterogeneity of early Parkinson's disease: a cluster analysis on newly diagnosed untreated patients. PLoS One. 2013;8(8):e70244.

8. Everitt B, Landau S, Leese M, Stahl D. Cluster analysis. Chichester Whiley, 2011.

9. Selikhova M, Williams DR, Kempster PA, Holton JL, Revesz T, Lees AJ. A clinico-pathological study of subtypes in Parkinson's disease. Brain. 2009;132(Pt 11):2947-57.

10. Nutt JG. Motor subtype in Parkinson's disease: Different disorders or different stages of disease? Mov Disord. 2016;31(7):957-61.

11. Mu J, Chaudhuri KR, Bielza C, de Pedro-Cuesta J, Larranaga P, Martínez-Martín P. Parkinson's disease subtypes identified from cluster analysis of motor and non-motor symptoms. Front Aging Neurosci. 2017;9:301.

12. Parkinson Progression Marker Initiative. The Parkinson Progression Marker Initiative (PPMI). Prog Neurobiol. 2011;95(4):629-35.

13. Cervantes-Arriaga A, Rodríguez-Violante M, López-Ruiz M, Estrada-BeIlmann I, Zuñiga-Ramírez C, Otero-Cerdeira E, et al. [Profile characterization of Parkinson's disease in Mexico: ReMePARK study]. Gac Med Mex. 2013;149(5):497-501.

14. Eisinger RS, Hess CW, Martínez-Ramírez D, et al. Motor subtype changes in early Parkinson's disease. Parkinsonism Relat Disord. 2017; 43:67-72. 